\title{
Investigation of carbapenem heteroresistance among different sequence types of Pseudomonas aeruginosa clinical isolates reveals further diversity
}

Pseudomonas aeruginosa is a leading infectious agent causing difficult-to-treat nosocomial infections due to the multiresistant phenotype that it often exhibits. Expression of the intrinsic AmpC $\beta$-lactamase, overexpression of Mex-Opr-type efflux pumps as well as repression of porin OprD have been associated with $\beta$-lactam resistance in this species (Lister et al., 2009). Also, different genotypes of carbapenem-susceptible $P$. aeruginosa have been documented to produce carbapenem-heteroresistant subpopulations (Pournaras et al., 2007).

Briefly, besides their elevated carbapenem MICs, the carbapenem-heteroresistant $P$. aeruginosa subpopulations were found to have a prolonged lag phase and a short exponential phase in the growth curve relative to their native populations (Pournaras et al., 2007). This might explain their delayed phenotypic detection, up to $48 \mathrm{~h}$ of incubation at $37{ }^{\circ} \mathrm{C}$ for some isolates, as distinct colonies within the inhibition zone of carbapenem Etests or discs. Also, the heterogeneous subpopulations were found to have the intrinsic efflux pump systems MexAB-OprM and MexXY-OprM upregulated while porin $\mathrm{OprD}$ was downregulated, relative to the native populations (Ikonomidis et al., 2008); the combination of these antimicrobial resistance mechanisms was suggested to contribute to carbapenem heteroresistance in $P$. aeruginosa.

This report investigates the distribution of carbapenem heteroresistance among several $P$. aeruginosa sequence types (STs) and documents the further diversity of this phenotype.

All single $P$. aeruginosa clinical isolates that were initially identified as susceptible to at least one carbapenem (imipenem or meropenem) by the VITEK 2 automated system (bioMérieux) in the Laboratory of
Microbiology, University Hospital of Larissa, Greece, were included in the study. The study period lasted from late November 2007 to early December 2008. MICs of imipenem, meropenem, piperacillin/tazobactam, ceftazidime, ciprofloxacin, amikacin and gentamicin were tested by agar dilution according to Clinical and Laboratory Standards Institute criteria (CLSI, 2008). Reference strain P. aeruginosa ATCC 27853 as well as previously characterized carbapenemheteroresistant $P$. aeruginosa clinical isolate 6315 (Pournaras et al., 2007) were used as controls. The genetic relationship of the tested isolates was investigated by PFGE of XbaI-digested genomic DNA; banding patterns were compared using criteria proposed by Tenover et al. (1995). Also, multilocus sequence typing (MLST) was performed according to a previously described scheme (Curran et al., 2004) and STs were assigned through the $P$. aeruginosa MLST database (http:// pubmlst.org/paeruginosa/).

Population analysis profile assays (PAPs) with imipenem and meropenem were performed by spreading approximately $10^{8}$ c.f.u. (100 $\mu \mathrm{l}$ of a starting inoculum containing approx. $10^{9}$ c.f.u. $\mathrm{ml}^{-1}$ ) on Mueller-Hinton agar plates with imipenem or meropenem in serial dilutions for concentrations ranging from 1 to $32 \mathrm{mg} \mathrm{l}^{-1}$ and incubating the plates for $48 \mathrm{~h}$. The analysis was performed three times for all isolates and mean values of viable c.f.u. were calculated. The frequency of appearance of heteroresistant subpopulations at the highest drug concentration was calculated by dividing the number of colonies grown on the antibiotic-containing plate by the colony counts from the same bacterial inoculum grown on antibiotic-free plates. For each strain, three distinct colonies grown at the highest drug concentration were subcultured daily in antibiotic-free medium for 1 week and carbapenem MICs were tested by agar dilution on Mueller-Hinton agar plates to check the stability of heteroresistant phenotypes.

During the study period, 283 P. aeruginosa clinical isolates were recorded from which 195 (68.9\%) were susceptible to imipenem and/or meropenem. Twenty-one of the latter isolates were selected at random and further investigated with respect to their putative heteroresistant phenotype. MICs of imipenem and meropenem ranged from $<1$ to $16 \mathrm{mg} \mathrm{l}^{-1}$ and from $<1$ to $32 \mathrm{mg}$ $1^{-1}$, respectively. The susceptibility to the remaining antipseudomonal antimicrobials is shown in Table 1. The isolates belonged to at least 15 distinct genotypes as shown by PFGE analysis, and 12 ST types as shown by MLST analysis. Of note, seven isolates belonged to ST696, which is a single locus variant of ST235, a member of international clonal complex CC11 (Libisch et al., 2008), which has been associated with European epidemic clones (Nemec et al., 2010).

In PAPs using imipenem, heteroresistant subpopulations showed two- to fourfoldhigher MICs versus the native populations when retested after subculture in the absence of antibiotics. Five isolates (23.81\%) did not exhibit imipenemheteroresistant subpopulations (PA124, PA140, PA226, PA243 and PA273) but two of them were meropenem heteroresistant (PA140 and PA273). In PAPs using meropenem, heteroresistant subpopulations showed two- to $>$ fourfold-higher MICs when retested after free-of-drug subcultures versus the native populations. Six isolates $(28.57 \%)$ did not exhibit meropenem-heteroresistant subpopulations (PA10, PA33, PA63, PA124, PA226 and PA243) but three of them were imipenem heteroresistant (PA10, PA33 and PA63). Overall, three out of the 21 tested isolates $(14.29 \%)$ did not produce stable heteroresistant 
Table 1. Characteristics of the isolates of the study

Differences of onefold between agar dilution and PAP were not evaluated. Numbers in bold and underlined refer to the cases in which the heterogeneous subpopulations returned to the parental phenotype. IMP, Imipenem; MEM, meropenem.

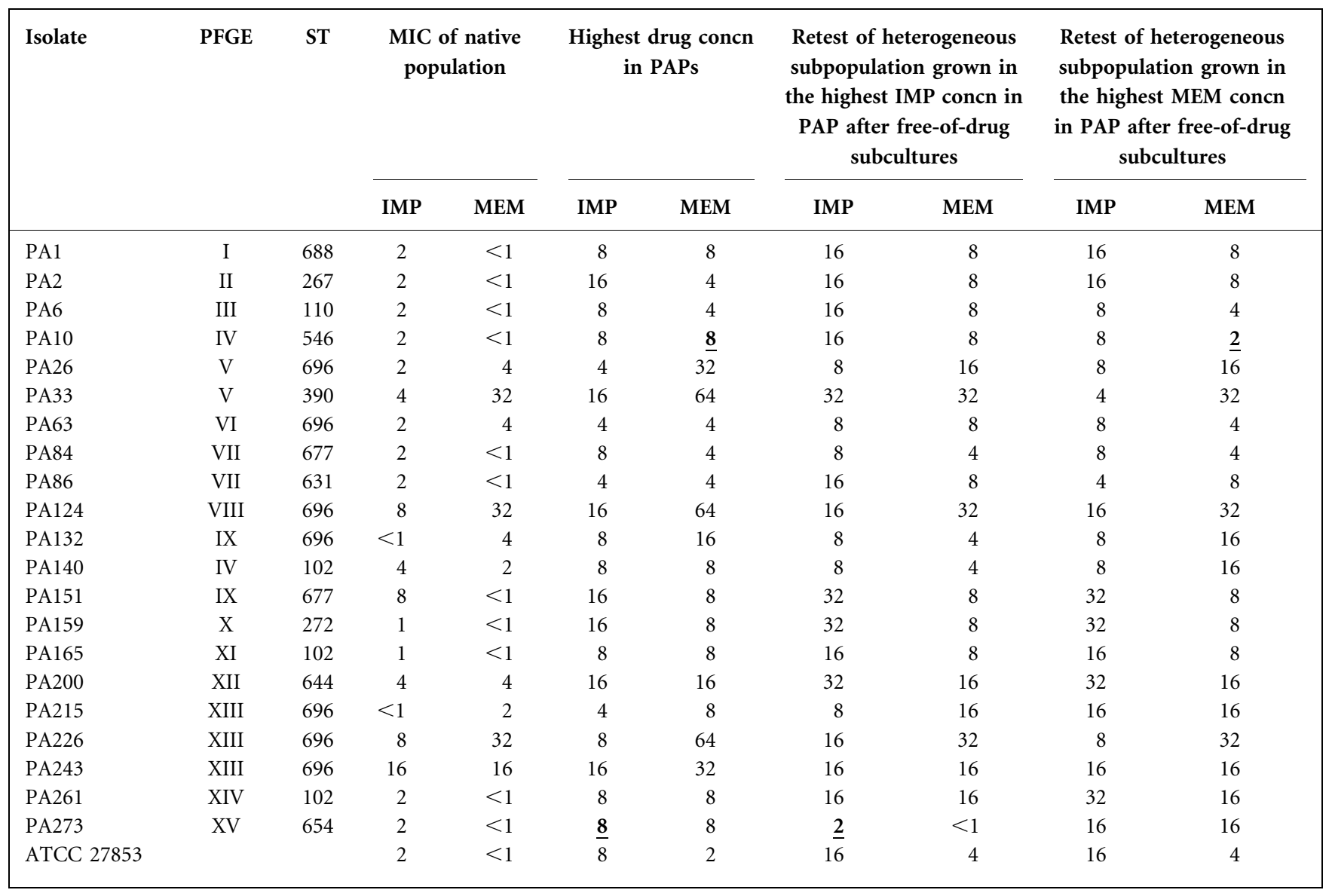

subpopulations (PA124, PA226 and PA243). In addition, in two isolates (PA10 and PA273) the heterogeneous subpopulations of meropenem and imipenem, respectively, returned to the parental phenotype. Interestingly, carbapenem-heteroresistant subpopulations showing more than twofold-higher carbapenem MICs were also recovered from reference strain ATCC 27853. This could be attributed to the higher inoculum used in this study relative to the original publication (Pournaras et al., 2007). The frequency of imipenemheteroresistant subpopulations ranged from $5 \times 10^{-7}$ to $10^{-2}$ and that of meropenemheteroresistant subpopulations ranged from $4 \times 10^{-7}$ to $2 \times 10^{-2}$.

Although there is not yet a formal definition of heteroresistance, it could be defined as resistance manifested by a fraction of a microbial population that is generally considered to be susceptible to the respective antimicrobial (Falagas et al., 2008). According to Balaban et al. (2004), a bacterial population might survive antibiotic treatment through resistant mutants which show stable higher MICs to the antimicrobial or through persistent cells that return to the parental phenotype when the antibiotic pressure is withdrawn. This report shows that $P$. aeruginosa might produce both types of heteroresistant subpopulations when a carbapenem is used. Even within the same genotype, for example genotype XIII, isolates might exhibit different phenotypic profiles when carbapenem is used, varying from homogeneous susceptibility (PA243) to production of heteroresistant subpopulations with stable higher MICs (PA215). Moreover, a subpopulation might survive antimicrobial pressure through both a fraction of persisters and a fraction of stable resistant mutants as shown by some isolates in our collection (data not shown).

Overall, although carbapenem heteroresistance seems a dispersed trait among $P$. aeruginosa, it appears diverse even within the same bacterial population. In addition to the detected phenotypic diversity it should be noted that the original observations on Mex-Opr-type efflux pumps and porin OprD

(Ikonomidis et al., 2008) were not replicated in all of the isolates of this study. Indicatively for strain ATCC 27853, imipenem and meropenem subpopulations showed a $+0.66( \pm 0.14)$ and $+0.36( \pm 0.16)$-fold change in gene mex $B$ expression respectively, relative to the native population, whereas expression of gene mexY showed no difference. Of interest, gene $\operatorname{oprD}$ showed a +0.83 $( \pm 0.20)$ - and $+1.07( \pm 0.15)$-fold change and $\mathrm{a}-0.35( \pm 0.07)$ - and -0.31 
$( \pm 0.11)$-fold change in gene mexE expression in imipenem and meropenem subpopulations, respectively. Similar observations were also made for other isolates (data not shown), which further complicates the issue.

\section{Acknowledgements}

This work was supported by a Research Grant from the European Society of Clinical Microbiology and Infectious Diseases received by the corresponding author, Dr Alexandros Ikonomidis, in 2009 (Project: Molecular investigation of carbapenem heteroresistance in Pseudomonas aeruginosa and in vivo evaluation of therapeutic options).

\section{Olga Oikonomou, ${ }^{1}$ Maria Panopoulou ${ }^{2}$ and Alexandros Ikonomidis ${ }^{1}$}

${ }^{1}$ Laboratory of Clinical Microbiology,

School of Medicine, University of

Thessaly, Larissa, Greece

${ }^{2}$ Laboratory of Clinical Microbiology, School of Medicine, Democritus University of Thrace, Alexandroupolis, Greece
Correspondence: Alexandros Ikonomidis (ado@med.uth.gr)

Balaban, N. Q., Merrin, J., Chait, R., Kowalik, L. \& Leibler, S. (2004). Bacterial persistence as a phenotypic switch. Science 305, 1622-1625.

CLSI (2008). Performance Standards for Antimicrobial Susceptibility Testing, 18th Informational Supplement. M100-S18. Wayne, PA: Clinical and Laboratory Standards Institute.

Curran, B., Jonas, D., Grundmann, H., Pitt, T. \& Dowson, C. G. (2004). Development of a multilocus sequence typing scheme for the opportunistic pathogen Pseudomonas aeruginosa. J Clin Microbiol 42, 56445649.

Falagas, M. E., Makris, G. C., Dimopoulos, G. \& Matthaiou, D. K. (2008). Heteroresistance: a concern of increasing clinical significance? Clin Microbiol Infect 14, 101-104.

Ikonomidis, A., Tsakris, A., Kantzanou, M., Spanakis, N., Maniatis, A. N. \& Pournaras, S. (2008). Efflux system overexpression and decreased OprD contribute to the carbapenem heterogeneity in Pseudomonas aeruginosa. FEMS Microbiol Lett 279, 36-39.

Libisch, B., Poirel, L., Lepsanovic, Z., Mirovic, V., Balogh, B., Pászti, J., Hunyadi, Z., Dobák, A., Füzi, M. \& Nordmann, P. (2008). Identification of PER-1 extended-spectrum beta-lactamase producing Pseudomonas aeruginosa clinical isolates of the international clonal complex CC11 from Hungary and Serbia. FEMS Immunol Med Microbiol 54, 330-338.

Lister, P. D., Wolter, D. J. \& Hanson, N. D. (2009). Antibacterial-resistant Pseudomonas aeruginosa: clinical impact and complex regulation of chromosomally encoded resistance mechanisms. Clin Microbiol Rev 22, 582-610.

Nemec, A., Krizova, L., Maixnerova, M. \& Musilek, M. (2010). Multidrug-resistant epidemic clones among bloodstream isolates of Pseudomonas aeruginosa in the Czech Republic. Res Microbiol 161, 234-242.

Pournaras, S., Ikonomidis, A., Markogiannakis, A., Spanakis, N., Maniatis, A. N. \& Tsakris, A. (2007). Characterization of clinical isolates of Pseudomonas aeruginosa heterogeneously resistant to carbapenems. J Med Microbiol 56, 66-70.

Tenover, F. C., Arbeit, R. D., Goering, R. V., Mickelsen, P. A., Murray, B. E., Persing, D. H. \& Swaminathan, B. (1995). Interpreting chromosomal DNA restriction patterns produced by pulsed-field gel electrophoresis: criteria for bacterial strain typing. J Clin Microbiol 33, 2233-2239. 